\title{
Changing trends in clinical characteristics and antibiotic susceptibility of Klebsiella pneumoniae bacteremia
}

Miri Hyun, Chang In Noh, Seong Yeol Ryu, and Hyun Ah Kim

Department of Infectious Diseases, Keimyung University Dongsan Medical Center, Daegu, Korea

Received: August 5, 2015

Revised : February 1, 2016

Accepted: January 12, 2017

\section{Correspondence to}

\section{Hyun Ah Kim, M.D.}

Department of Infectious Diseases, Keimyung University Dongsan Medical Center, 56 Dalseongro, Jung-gu, Daegu 41931, Korea

Tel: $+82-53-250-7892$

Fax: $+82-53-250-7434$

E-mail: hyunah1118@hanmail.net
Background/Aims: Klebsiella pneumoniae is second most common organism of gram-negative bacteremia in Korea and one of the most common cause of urinary tract infection, and intra-abdominal infection.

Methods: We compared clinical and microbiological characteristics about K. pneumoniae bacteremia in a tertiary hospital between 10 years. Group A is who had $K$. pneumoniae bacteremia at least one time from January 2004 to December 2005. Group B is from January 2012 to December 2013. We also analyzed antibiotic resistance, clinical manifestation of the $\mathrm{K}$. pneumoniae bacteremia divided into community-acquired infections, healthcare associated infections, and nosocomial infections.

Results: The resistance for ampicillin, aztreonam, cefazolin, and cefotaxime significantly increased compared to 10 years ago. Extended spectrum $\beta$-lactamase positivity surged from $4.3 \%$ to $19.6 \%$. Ten years ago, 1st, 2nd cephalosporin, and aminoglycoside were used more as empirical antibiotics. But these days, empirical antibiotics were broad spectrum such as 3 rd and 4 th cephalosporin. In treatment outcome, acute kidney injury decreased from $47.5 \%$ to $28.7 \%$, and mortality decreased from $48.9 \%$ to $33.2 \%$. In community-acquired infections, there was similar in antimicrobial resistance and mortality. In healthcare-associated and nosocomial infections, there was significantly increasing in antibiotic resistance, decreasing in mortality, and acute kidney injury.

Conclusions: In community-acquired infections, broader antibiotics were more used than 10 years ago despite of similar antimicrobial resistance. When K. pneumoniae bacteremia is suspected, we recommend to use the narrow spectrum antibiotics as initial therapy if there are no healthcare-associated risk factors, because the antibiotic resistance is similar to 10 years ago in community-acquired infections.

Keywords: Klebsiella pneumoniae; Bacteremia; Drug resistance

\section{INTRODUCTION}

Klebsiella pneumoniae is a gram-negative rod-shaped bacteria that can cause urinary tract infection, pneumonia, and intra-abdominal infection. $K$. pneumoniae is the main cause of hepatobiliary infection in community-acquired infections and urinary tract infection, pneumonia in healthcare-associated infections and nosocomial 
infections [1,2].

$K$. pneumoniae is the second most common gram-negative bacteria, following to Escherichia coli, and one of the most important bacteria of mortality and morbidity. It has been the most common cause of liver abscess in East Asia since the 1990s. The association of K. pneumoniae and liver abscess in Korea has been known since the 1990 [1].

Recently, antimicrobial resistance of gram-negative bacteria has been gradually increased worldwide [3]. According to a report of South Korea, the prevalence of $K$. pneumoniae which resistant to ciprofloxacin was increased from $17.1 \%$ in 2006 to $26.5 \%$ in 2011 , and the proportion of multidrug-resistant $K$. pneumoniae producing extended spectrum $\beta$-lactamase (ESBL) increased from $13.6 \%$ in 2006 to 2007 to $28.5 \%$ in 2011 [4].

The aim of this study is to find out the trends of $K$. pneumoniae bacteremia during the last 10-year period. These study groups were divided into two groups as 2004 to 2005 and 2012 to 2013 , and analyzed differences about focuses of bacteremia, antibiotic resistance, empirical antibiotics, adequacies, and treatment responses. We also analyzed separately two groups for communityacquired infection and healthcare-associated infection/ nosocomial infection.

\section{METHODS}

\section{Study subjects}

Patients who visited Keimyung University Dongsan Medical Center and had K. pneumoniae bacteremia were divided into two groups, as group A was between January 2004 and December 2005 and group B was between January 2012 and December 2013, respectively. Patients under 18 years old or polymicrobial bacteremia were excluded. Patients who were transferred to other hospitals during treatment were also excluded. There were the ${ }_{169} \mathrm{~K}$. pneumoniae bacteremia cases in group A; eight patients were excluded for the polymicrobial bacteremia; nine children were excluded; and 12 patients were excluded for transferred to other hospitals. There were the $230 \mathrm{~K}$. pneumoniae bacteremia cases in group B; four patients were excluded for the polymicrobial bacteremia; six children were excluded; and 16 patients were excluded for transferred to other hospitals (Fig. 1). One hundred and forty patients in group A and 204 patients in group B were retrospectively investigated.

\section{Study item}

Medical records, including underlying diseases, previous antibiotics, previous admissions of intensive care unit (ICU), antibiotic resistance rates, clinical features, and treatments, were retrospectively analyzed. Age and sex in two groups were compared; antibiotics resistance rates and empirical antibiotics were compared and analyzed by each group. The focus of infection was determined according to physician's diagnosis based on patient symptoms, physical examination findings, and detections of $K$. pneumoniae in other samples.

Community-acquired infections were defined as those in which symptoms occurred within 48 hours after visiting the hospital. Nosocomial infections were defined as those in which symptoms occurred 48 hours after hos-
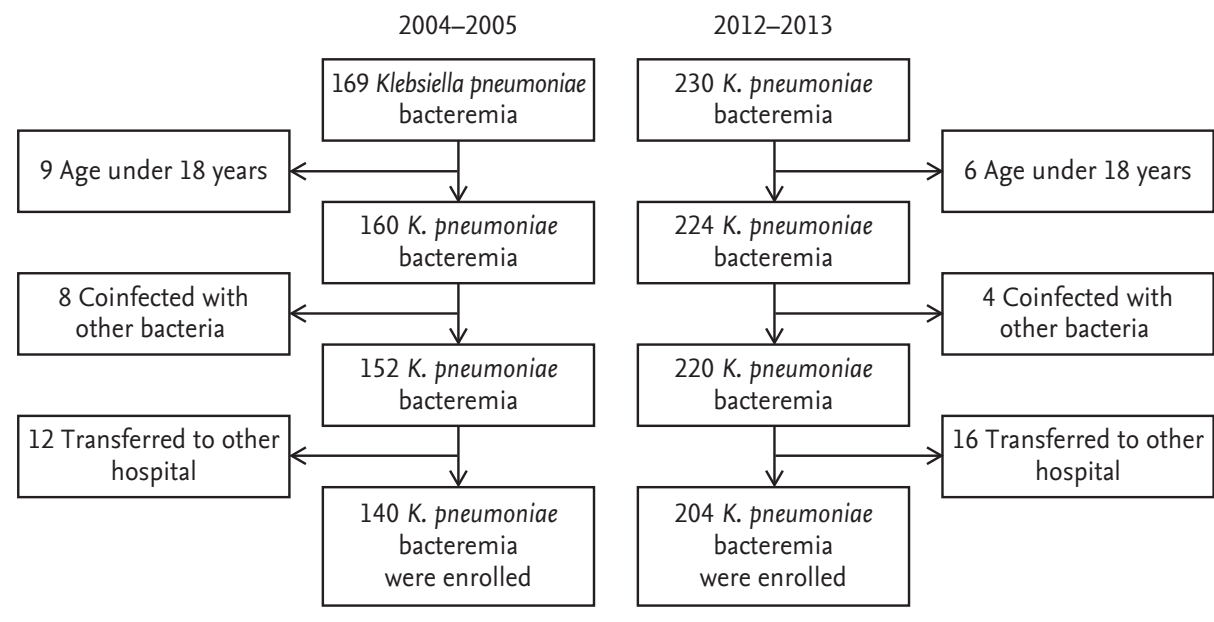

Figure 1. Enrollment of patients in the study. 
pital admission. Patients with community-acquired infections who had healthcare-associated risk factors were categorized as healthcare-associated infections. Healthcare-associated risk factors were hospitalization within 90 days, received dialysis, taken intravenous medication in outpatient clinics, or resided in long-term care facilities.

Pitt bacteremia score was calculated based on temperature $\left(35.1^{\circ} \mathrm{C}\right.$ to $36^{\circ} \mathrm{C}$ or $39.0^{\circ} \mathrm{C}$ to $39.9^{\circ} \mathrm{C}$, 1 point; s $35^{\circ} \mathrm{C}$ or $\geq 40^{\circ} \mathrm{C}, 2$ points), blood pressure (hypotension, 2 points), mental status (disorientation, 1 point; stupor, 2 points; coma, 4 points), respiratory status (mechanical ventilation, 2 points), and cardiac status (cardiac arrest, 4 points). The worst reading was recorded on the day the first positive blood culture was obtained or the day before for nosocomial bloodstream infections.

Treatment outcome was evaluated after 72 hours of empirical antibiotics, based on infection-related mortality, admission of ICU, acute kidney injury, mechanical ventilation, recurrence of bacteremia, and laboratory tests. Treatment outcome was classified as complete response, partial response, and treatment failure. Complete response was defined as both laboratory findings and clinical states were improved. Partial response was defined as either laboratory findings or clinical states was improved. Treatment failure was defined as both were worsened and infection-related death. If patient died of K. pneumoniae bacteremia or complication of infection, we defined this situation as infection-related death. Acute kidney injury was defined as increase in serum creatinine by $>0.3$ $\mathrm{mg} / \mathrm{dL}$ within 48 hours; or increase in serum creatinine to $>1.5$ times baseline, which have occurred within the prior 7 days; or urine volume $<0.5 \mathrm{~mL} / \mathrm{kg} / \mathrm{hr}$ for 6 hours.

\section{Statistics}

Statistical analysis was performed using SPSS version 21.o (IBM Co., Armonk, NY, USA) and MedCalc for Windows version 16.4.1. (MedCalc Software, Ostend, Belgium). Binary data were compared using the chi-square test and continuous scaled analysis were compared using Student $t$ test. The data were confirmed to have a normal distribution; mean values were used as representative values. Statistical significance was defined as $p$ values less than 0.05 and Kaplan-Meier survival analysis was used to test for correlations between clinical features, mortality, and hazard ratio. This study was approved by the Ethical Committees and Institutional Review Boards of Keimyung University Dongsan Medical Center, Daegu, Korea (IRB No. 2014-02-008). IRB approved a waiver of the requirements to obtain informed consent for this study.

\section{RESULTS}

\section{Basic characteristics of study group}

In group A, 140 patients were included; 86 (61.4\%) were males; and the mean age was 68.5 years. In group B, 204 patients were included; 116 (56.9\%) were males; and the mean age was 68.0 years. There were no statistically significant differences in gender or age between two groups.

Analysis of underlying diseases revealed that group B had significantly higher incidence of cardiovascular and cerebrovascular disease than group A. Incidence rates of community-acquired, healthcare-associated infections and nosocomial infections were each $53 \%, 20.1 \%$, and $26.6 \%$ in group A, and $45.6 \%, 27.5 \%$, and $27 \%$ in group B, respectively (Table 1). Mean Pitt score was 4.81 in group A and 2.93 in group B and that was statistically significant. In community-acquired infection, Pitt score was 4.15 in group A and 3.41 in group B. In healthcare-associated infection/nosocomial infections, Pitt score was 5.43 in group A and 2.53 in group B.

In community-acquired infections, hepatobiliary infections were most prevalent, $43.2 \%$ and $48.4 \%$ each in group A and B. In nosocomial infections, pneumonia was the most prevalent with rates of $16.7 \%$ and $24.3 \%$ each in group A and B, followed by hepatobiliary infections, with rates of $21.2 \%$ and $17.1 \%$ each in group $A$ and $\mathrm{B}$, but this difference between group $\mathrm{A}$ and $\mathrm{B}$ was not statistically significant.

\section{Comparison of antibiotic resistance, empirical anti- biotics, and antibiotic adequacy}

Previous antibiotics within 1 month had increased significantly compared to 10 years before (7.9\% vs. $27.1 \%$, $p$ $=0.001$ ); however, the types of antibiotics did not differ significantly.

Antibiotic resistance had increased between group A and B significantly. Rates of ESBL-producing bacteria, and resistance to aztreonam, cefotaxime, and cefepime 
Table 1. Comparison of Klebsiella pneumoniae bacteremia according to sex and category of infection

\begin{tabular}{|c|c|c|c|}
\hline Variable & ${\text { Group } A^{a}(n=140)}$ & ${\text { Group } B^{b}(n=204)}^{b}$ & $p$ value \\
\hline Age, yr & $68.5 \pm 11$ & $68 \pm 14$ & 0.308 \\
\hline Sex & & & 0.436 \\
\hline Male & $86(61.4)$ & $116(56.9)$ & \\
\hline Female & $54(38.6)$ & $88(43.1)$ & \\
\hline Category of infection & & & 0.155 \\
\hline Community-acquired & $75(53.2)$ & $93(45.6)$ & \\
\hline Healthcare-associated & $25(20.1)$ & $56(27.5)$ & \\
\hline Nosocomial & $37(26.6)$ & $55(27.0)$ & \\
\hline \multicolumn{4}{|l|}{ Comorbidity } \\
\hline Solid tumor & $43(30.7)$ & $73(36.1)$ & 0.353 \\
\hline Hematologic malignancy & $6(4 \cdot 3)$ & $15(7 \cdot 4)$ & 0.261 \\
\hline Cardiovascular disease & $34(24 \cdot 3)$ & $96(47.5)$ & 0.001 \\
\hline Neurovascular disease & $15(10.8)$ & $40(19.8)$ & 0.035 \\
\hline Diabetes mellitus & $48(34 \cdot 3)$ & $75(37.1)$ & 0.647 \\
\hline \multicolumn{4}{|l|}{ Origin of infection } \\
\hline Hepatobiliary infection & $46(32.9)$ & $64(31.4)$ & 0.814 \\
\hline Urinary tract infection & $24(17.1)$ & $35(17.2)$ & 1.000 \\
\hline Respiratory infection & $21(15.0)$ & $34(16.7)$ & 0.765 \\
\hline Intra-abdominal infection & $14(10.0)$ & $20(9.8)$ & 1.000 \\
\hline Surgical site infection & $3(2.1)$ & $3(1.5)$ & 0.691 \\
\hline Catheter related infection & $1(0.7)$ & $8(3 \cdot 9)$ & 0.089 \\
\hline CNS infection & $1(0.7)$ & $1(0.5)$ & 1.000 \\
\hline Bone/joint infection & $\mathrm{O}$ & $2(1.0)$ & 0.516 \\
\hline
\end{tabular}

Values are presented as mean \pm SD or number (\%).

CNS, central nervous system.

${ }^{\mathrm{a}}$ Group A, 2004-2005. ' Group B, 2012-2013.

increased significantly over time (Table 2). Antibiotic resistance was analyzed separately for communityacquired, and healthcare-associated infections/nosocomial infections. It showed no differences in community-acquired infections between group A and B. However, antibiotic resistance for healthcare-associated infections/nosocomial infections increased significantly compared to 10 years previously (Table 3 ).

Ten years before, second-generation cephalosporin and aminoglycoside were most commonly used as empirical antibiotics. Recently, broad-spectrum antibiotics such as fourth-generation cephalosporin, carbapenem, and vancomycin were commonly used as empirical antibiotics and it increased significantly (Fig. 2). In analysis of empirical antibiotics, broad-spectrum antibiotics had increased, such as fourth-generation cephalosporin and carbapenem, especially in community-acquired infections (Table 4).

Comparison of adequacy of empirical antibiotics according to antibiotic susceptibility showed that use of broad-spectrum antibiotics compared with antimicrobial resistance increased significantly compared to past ( $5.0 \%$ vs. $28.5 \%, p=0.001$ ).

\section{Comparison of treatment outcomes}

Assessment of treatment outcome after 72 hours of management revealed improvement in group B. Complete response was in $3.6 \%$ and $25.2 \%$ of group A and group B patients, partial response in $59.7 \%$ and $40.6 \%$, and treatment failure in $31.6 \%$ and $34.2 \%$. Rates of acute kidney injury, mechanical ventilation, and 30-day mortality were decreased in group B, significantly (Table 5). 
Table 2. Comparison of antibiotic resistance

\begin{tabular}{lccc}
\hline Variable & Group A $^{\mathrm{a}}$ & Group B $^{\mathrm{b}}$ & p value \\
\hline Amikacin & $10(7.1)$ & $10(4.9)$ & 0.483 \\
Amoxicillin/clavulanate & $16(11.4)$ & $18(8.8)$ & 0.603 \\
Ampicillin & $135(96.4)$ & $204(100)$ & 0.025 \\
Aztreonam & $13(9.3)$ & $41(20.1)$ & 0.013 \\
Cefazolin & $14(10.0)$ & $43(21.1)$ & 0.013 \\
Cefepime & 0 & $39(19.1)$ & 0.001 \\
Cefotaxime & $10(7.1)$ & $41(20.1)$ & 0.001 \\
Cefoxitin & $7(5.0)$ & $14(6.9)$ & 0.729 \\
Ciprofloxacin & $9(6.4)$ & $24(11.8)$ & 0.113 \\
Gentamicin & $13(9.3)$ & $27(13.2)$ & 0.503 \\
Piperacillin/tazobactam & $10(7.2)$ & $20(9.8)$ & 0.344 \\
Tigecycline & $8(5.7)$ & $9(4.4)$ & 0.706 \\
TMP/SMX & $12(8.6)$ & $26(12.7)$ & 0.237 \\
ESBL positivity & $6(4.3)$ & $40(19.6)$ & 0.001 \\
\hline
\end{tabular}

Values are presented as number (\%).

TMP, trimethoprim; SMX sulfamethoxazole; ESBL, extended-spectrum $\beta$-lactam.

${ }^{\mathrm{a}}$ Group A, 2004-2005. ${ }^{\mathrm{b}}$ Group B, 2012-2013.
In healthcare-associated infection/nosocomial infection, overall treatment outcome has improved. Specifically, complete response increased in group B compared to group A, and the treatment failure, 30-day mortality, and mechanical ventilation was decreased in group B compared to group A. They were also significantly reduced (Table 6).

In community-acquired infection, treatment outcome was improved overall. Complete response was observed in $25 \%$ of patients in group B compared to $5.5 \%$ in group A, while treatment failure increased to $31.5 \%$ in group B from $30.1 \%$ in group A. Differences between the occurrence rates of two groups of acute kidney injury, mechanical ventilation, and 30-day mortality were not statistically significant (Table 6).

Kaplan-Meier survival analysis was performed to recognize the difference in survival rates between group A and B (Fig. 3). In group B, survival rates increased significantly compared to group A (hazard ratio, 1.53; 95\% CI, 1.08 to $2.18 ; p=0.011$ ).

Table 3. Comparison of antibiotic resistance in community-acquired infection and healthcare associated infection/nosocomial infection between two groups

\begin{tabular}{|c|c|c|c|c|c|c|}
\hline \multirow{2}{*}{ Variable } & \multicolumn{3}{|c|}{ Community-acquired infection } & \multicolumn{3}{|c|}{ Healthcare-associated/nosocomial infection } \\
\hline & Group $A^{a}(n=74)$ & Group $B^{b}(n=93)$ & $p$ value & ${\text { Group } A^{a}(n=66)}$ & Group $B^{b}(n=111)$ & $p$ value \\
\hline Amikacin & $2(2.7)$ & $1(1.1)$ & 0.585 & $8(12.1)$ & $9(8.1)$ & 0.433 \\
\hline Amoxicillin/clavulanate & $5(6.8)$ & $1(1.1)$ & 0.033 & $11(16.7)$ & $17(15 \cdot 3)$ & 0.851 \\
\hline Ampicillin & $70(94.6)$ & $93(100)$ & 0.076 & $65(98.5)$ & $111(100)$ & 0.373 \\
\hline Aztreonam & $5(6.8)$ & $5(5 \cdot 4)$ & 0.752 & $8(12.1)$ & $36(32.4)$ & 0.005 \\
\hline Cefazolin & $5(6.8)$ & $6(6.5)$ & 1.000 & $9(13.6)$ & $37(33 \cdot 3)$ & 0.008 \\
\hline Cefepime & 0 & $5(5 \cdot 4)$ & 0.067 & 0 & $34(30.6)$ & 0.001 \\
\hline Cefotaxime & $3(4.1)$ & $5(5 \cdot 4)$ & 0.495 & $7(10.6)$ & $36(32.4)$ & 0.002 \\
\hline Cefoxitin & $3(4 \cdot 1)$ & $2(2.2)$ & 0.406 & $4(6.1)$ & $12(10.8)$ & 0.557 \\
\hline Ceftazidime & $3(4 \cdot 1)$ & $5(5 \cdot 4)$ & 0.495 & $5(7 \cdot 6)$ & $35(31.5)$ & 0.001 \\
\hline Ciprofloxacin & $2(2.7)$ & $2(2.2)$ & 1.000 & $7(10.6)$ & $22(19.8)$ & 0.135 \\
\hline Gentamicin & $5(6.8)$ & $2(2.2)$ & 0.174 & $8(12.1)$ & $25(22.5)$ & 0.221 \\
\hline Piperacillin/tazobactam & $2(2.7)$ & $2(2.2)$ & 0.970 & $8(12.1)$ & $18(16.2)$ & 0.165 \\
\hline Tigecycline & $4(5 \cdot 4)$ & $1(1.1)$ & 0.259 & $4(6.1)$ & $8(7.2)$ & 0.520 \\
\hline TMP/SMX & $3(4 \cdot 1)$ & $3(3.2)$ & 1.000 & $9(13.6)$ & $23(20.7)$ & 0.224 \\
\hline ESBL positivity & $2(2.7)$ & $5(5 \cdot 4)$ & 0.465 & $4(6.1)$ & $35(31.5)$ & 0.001 \\
\hline
\end{tabular}

Values are presented as number (\%).

TMP, trimethoprim; SMX, sulfamethoxazole; ESBL, extended-spectrum $\beta$-lactam.

${ }^{\mathrm{a}}$ Group A, 2004-2005. ${ }^{\mathrm{b}}$ Group B, 2012-2013. 


\section{DISCUSSION}

There have been reports of community-acquired highly pathogenic strains of $K$. pneumoniae infections in South Korea. Hypervirulent $\mathrm{K} 1$ and $\mathrm{K} 2$ serotypes of K. pneumoniae were first identified as important causes of liver abscess in community-acquired infections since the 1990 s [5]. But, investigations of overall K. pneumoniae bacteremia are insufficient in South Korea. Therefore, this study was aim to analyze focuses of $K$. pneumoniae bacteremia, antimicrobial resistance, clinical characteristics, and risk factors among patients who visited a tertiary medical center between 2003 to 2004 (group A) and 2012 to 2013 (group B), retrospectively.

In this study, liver abscess and other hepatobiliary infection, urinary tract infection, and pneumonia were frequent cause of $K$. pneumoniae bacteremia, but not statistically significant. Liver abscess was most prevalent,

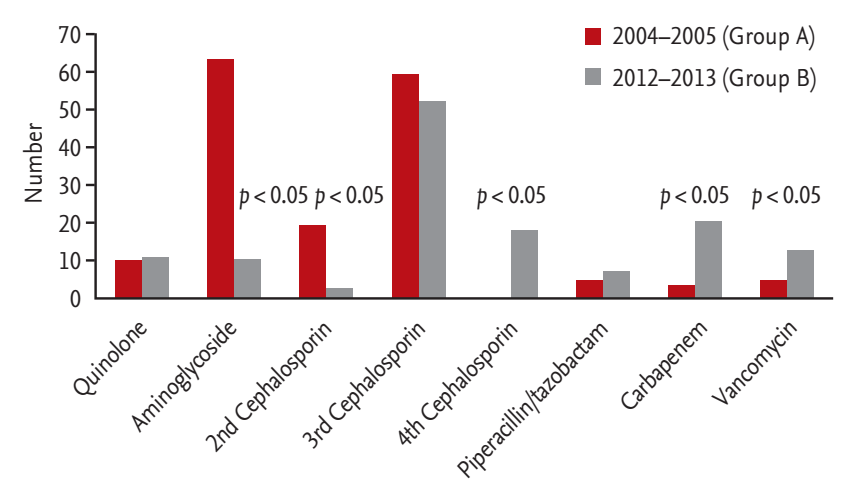

Figure 2. Comparison of empirical antibiotics. followed by urinary tract infections in community-acquired infections, while pneumonia and urinary tract infections were most prevalent in healthcare-associated infections and nosocomial infections. These findings are in accordance with other investigations conducted in South Korea as well as overseas. Other South Korean studies have reported that intra-abdominal infections and urinary tract infections to be the most prevalent cause of community-acquired $K$. pneumoniae bacteremia during the same period; intra-abdominal infection, pneumonia, and urinary tract infection were the most prevalent causes of nosocomial $K$. pneumoniae bacteremia $[6,7]$. In a study conducted overseas, liver abscess was prevalent in community-acquired infections, whereas pneumonia and catheter-associated infection were common in nosocomial infections [8].

Worldwide, the antibiotic resistance rates of gramnegative bacteria, including $\mathrm{K}$. pneumoniae, are gradually increasing [4]. This study found that recent antibiotic resistance of $\mathrm{K}$. pneumoniae in healthcare-associated infections and nosocomial infections was significantly increased compared to 10 years before. In subgroup analysis, antibiotic resistance was similar in community-acquired infection, and the recent increased antibiotic resistance was due to increased resistance in healthcare-associated infections and nosocomial infections. We found that antibiotics susceptibility of communityacquired $K$. pneumoniae bacteremia has been maintained for 10 years.

A study in South Korea showed that the rates of resistance to third-generation cephalosporin in community-

Table 4. Empirical antibiotics in community-acquired infection and healthcare associated infection/nosocomial infection between two groups

\begin{tabular}{|c|c|c|c|c|c|c|}
\hline \multirow{2}{*}{ Variable } & \multicolumn{3}{|c|}{ Community-acquired infection } & \multicolumn{3}{|c|}{ Healthcare-associated/nosocomial infection } \\
\hline & Group $A^{a}(n=74)$ & Group $B^{b}(n=93)$ & $p$ value & Group $A^{a}(n=66)$ & Group $B^{b}(n=111)$ & $p$ value \\
\hline 1st, 2nd cephalosporin & $16(21.9)$ & $1(1.1)$ & 0.001 & $10(15.2)$ & $3(2.7)$ & 0.005 \\
\hline 3rd cephalosporin & $43(58.9)$ & $59(64.1)$ & 0.522 & $39(59.1)$ & $46(41.8)$ & 0.030 \\
\hline 4th cephalosporin & o & $14(15.2)$ & 0.001 & 0 & $22(20.2)$ & 0.001 \\
\hline Aminoglycoside & $48(65.8)$ & $5(5 \cdot 4)$ & 0.001 & $40(60.6)$ & $15(13.6)$ & 0.001 \\
\hline Quinolone & $9(12.3)$ & $5(5 \cdot 4)$ & 0.160 & $4(6.1)$ & $16(14 \cdot 5)$ & 0.139 \\
\hline Piperacillin/tazobactam & $2(2.7)$ & $2(2.2)$ & 1.000 & $4(6.1)$ & $12(10.9)$ & 0.417 \\
\hline Imipenem/meropenem & o & $17(18.5)$ & 0.001 & $4(6.1)$ & $23(20.9)$ & 0.009 \\
\hline
\end{tabular}

Values are presented as number (\%).

${ }^{\mathrm{a}}$ Group A, 2004-2005. ${ }^{\mathrm{b}}$ Group B, 2012-2013. 
Table 5. Comparison of treatment response

\begin{tabular}{lccc}
\hline Variable & $\begin{array}{c}\text { Group } \mathrm{A}^{\mathrm{a}} \\
(\mathrm{n}=140)\end{array}$ & $\begin{array}{c}\text { Group B } \\
(\mathrm{n}=204)\end{array}$ & p value \\
\hline Response after 72 hr & & & 0.001 \\
\hline Complete response & $5(3.6)$ & $51(25.2)$ & \\
\hline Partial response & $83(59.7)$ & $82(40.6)$ & \\
\hline Failure & $51(31.6)$ & $69(34.2)$ & \\
ICU care & $54(38.8)$ & $65(32.2)$ & 0.206 \\
AKI & $66(47.5)$ & $58(28.7)$ & 0.001 \\
Mechanical ventilation & $41(29.5)$ & $34(16.8)$ & 0.008 \\
30-Day mortality & $68(48.9)$ & $66(33.2)$ & 0.005 \\
\hline
\end{tabular}

Values are presented as number (\%).

ICU, intensive care unit; AKI, acute kidney injury.

${ }^{\mathrm{a}}$ Group A, 2004-2005. ${ }^{\mathrm{b}}$ Group B, 2012-2013. acquired $E$. coli or $K$. pneumoniae infections increased from $6.1 \%$ in 2003 to 2008 to $10.6 \%$ in 2009 . In ICU patients with gram-negative bacteremia, ciprofloxacin was the most adequate antibiotic in 1999 [9]. In 2005, however, imipenem and tobramycin were reported adequate antibiotics rather than ciprofloxacin [10]. This result shows antibiotic resistance of gram-negative bacteria in ICU has been increased.

This study compared community-acquired infections and healthcare-associated infections/nosocomial infections during the same period, and found that healthcare-associated infections/nosocomial infections had higher mortality, although the difference was reduced compared to 10 years previously.

Table 6. Comparison of treatment response in community-acquired infection and healthcare associated infection/nosocomial infection between two groups

\begin{tabular}{|c|c|c|c|c|c|c|}
\hline \multirow{2}{*}{ Variable } & \multicolumn{3}{|c|}{ Community-acquired infection } & \multicolumn{3}{|c|}{ Healthcare-associated/nosocomial infection } \\
\hline & Group $A^{a}(n=74)$ & Group $B^{b}(n=93)$ & $p$ value & Group $A^{a}(n=66)$ & Group $B^{b}(n=111)$ & $p$ value \\
\hline Response after $72 \mathrm{hr}$ & & & 0.002 & & & 0.001 \\
\hline Complete response & $4(5 \cdot 5)$ & $23(25.0)$ & & $1(1.5)$ & $28(25 \cdot 5)$ & \\
\hline Partial response & $47(64.4)$ & $40(43.5)$ & & $36(54 \cdot 5)$ & $42(38.1)$ & \\
\hline Failure & $22(30.1)$ & $29(31.5)$ & & $29(43.9)$ & $40(36.4)$ & \\
\hline ICU care & $27(37.0)$ & $33(35.9)$ & 1.000 & $27(40.9)$ & $32(29.1)$ & 0.138 \\
\hline AKI & $26(35.6)$ & $23(30.4)$ & 0.508 & $40(60.6)$ & $30(27 \cdot 3)$ & 0.001 \\
\hline Mechanical ventilation & $22(30.1)$ & $18(19.6)$ & 0.144 & $19(28.8)$ & $16(14 \cdot 5)$ & 0.031 \\
\hline 30-Day mortality & $28(38.4)$ & $25(28.1)$ & 0.181 & $40(60.6)$ & $41(37 \cdot 3)$ & 0.003 \\
\hline
\end{tabular}

Values are presented as number (\%).

ICU, intensive care unit; AKI, acute kidney injury.

${ }^{\mathrm{a}}$ Group A, 2004-2005. ${ }^{\mathrm{b}}$ Group B, 2012-2013.

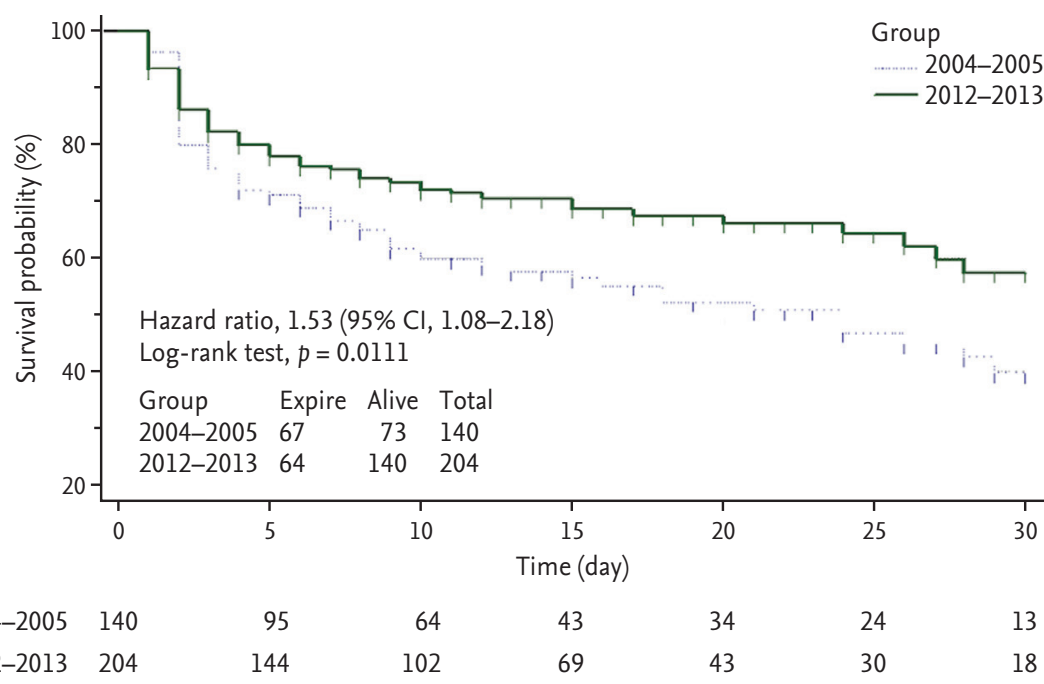
Group: 2004-2005 140 Group: 2012-2013 204

$144 \quad 102$

69
30
18 survival between two groups. 
A study by Kang et al. [1] reported that nosocomial K. pneumoniae infections had a $32.3 \%$ of 30 -day mortality, significantly higher than $16.2 \%$ for communityacquired infections. Factors influencing on mortality included inadequate empirical antibiotics, inadequate susceptible antibiotics, admission to ICU, septic shock, neutropenia, and use of immunosuppressants [11].

In this study, treatment outcome after 72 hours showed better and 30-day mortality was reduced compared to 10 years previously. Pitt score was decreased compared to 10 years previously, and it was statistically significant. Sepsis treatment guidelines are continuously changed; recent guideline suggested that providing sufficient fluids within the first 6 hours and prompt use of vasopressors in order to maintain central venous pressure of 8 to12 $\mathrm{mmHg}$, mean arterial pressure of $60 \mathrm{mmHg}$, and hourly urine volume of $0.5 \mathrm{~mL} / \mathrm{kg}$ to achieve early treatment in patients with suspected sepsis [12]. This practice may have improved treatment response within the first 72 hours and reduced acute kidney injury due to infection or ischemia which sometimes accompanies sepsis [9].

Morbidity and mortality of K. pneumoniae bacteremia are gradually increasing, according to a South Korean study that analyzed 147 patients with gram-negative bacteremia. K. pneumoniae bacteremia was $5.4 \%$ of gram-negative bacteremia, and its mortality was $37.5 \%$, higher mortality than other gram-negative bacterial infections [3,9,13].

This study revealed that in community-acquired infection, Pitt score was decreased from 4.15 to 3.41 between 10 years and antimicrobial resistance was similar, but use of broad-spectrum antibiotics increased from $4.1 \%$ to $35.5 \%$. According to these results, there was no change of antimicrobial resistance in community-acquired infections between two groups. It means that if $\mathrm{K}$. pneumoniae bacteremia is suspicious and healthcareassociated infection is excluded, excessive use of broadspectrum antibiotics in the early stage of disease may be reduced. Excessive use of broad-spectrum antibiotics makes more antibiotic resistance [14]. Therefore, adequate use of empirical antibiotics in communityacquired infection will help prevent antimicrobial resistance and reduce medical costs from inadequate use of expensive broad-spectrum antibiotics. In group A, $12 \%$ were inappropriate for initial empirical antibiotic and in group B, $15 \%$ were in appropriate. That was not statistically significant. Among cases of inappropriate empirical antibiotics, $5 \%$ was changed to definite antibiotics in group A, and $37.3 \%$ of group B was changed to definite antibiotics after identifying antibiotic susceptibility results. The change of the appropriate antibiotic might have influenced the mortality. However, antibiotic resistance dramatically increased among healthcareassociated infections and nosocomial infections. Use of broad-spectrum antibiotics in early stage may be considered for patients with risk factors of nosocomial or healthcare-associated infections.

This study was conducted in order to announce that broad-spectrum antibiotics were increased steadily despite of similar antimicrobial resistance in community-acquired K. pneumoniae bacteremia compared to 10 years before. Therefore, we agitate use of narrow spectrum antibiotics, if the patient has community-acquired Klebsiella bacteremia and doesn't have risk factors of healthcare-associated bacterial infection. We intent to announce in this study when community-acquired $\mathrm{K}$. pneumoniae bacteremia is suspected, like liver abscess or urinary tract infection, clinicians may need to reduce the use of broad spectrum antibiotics as initial treatment.

There are several potential limitations in this study. Firstly, this study was retrospective, conducted in a tertiary hospital, and relied on microbiological culture results, which may introduce bias in the data interpretation. Secondly, we should acknowledge that the patients included in this study were in a tertiary hospital and might be more severe than primary medical center. Despite of these limitations, we discovered the trends about antimicrobial resistance and pattern of antimicrobial prescription of $K$. pneumoniae bacteremia over 10 years.

\section{KEY MESSAGE}

1. This study showed that the overall antibiotic resistance rate of Klebsiella pneumoniae increased significantly compared to 10 years previously.

2. In community-acquired infections, the antibiotic resistance was not increased and Pitt bacteremia score was decreased than 10 years previously.

3. For both community-acquired and healthcare-associated infections, broad-spectrum antibiotics are more commonly used for empirical 
treatment compared to 10 years previously.

4. Unnecessary use of broad spectrum antibiotics can be reduced if $\mathrm{K}$. pneumoniae bacteremia is suspicious and there are no healthcare risk factors, because antibiotic resistance in community-acquired K. pneumoniae bacteremia was not increased.

\section{Conflict of interest}

No potential conflict of interest relevant to this article was reported.

\section{REFERENCES}

1. Kang CI, Kim SH, Bang JW, et al. Community-acquired versus nosocomial Klebsiella pneumoniae bacteremia: clinical features, treatment outcomes, and clinical implication of antimicrobial resistance. J Korean Med Sci 2006;21:816-822.

2. Sun HY, Chen SY, Chang SC, Pan SC, Su CP, Chen YC. Community-onset Escherichia coli and Klebsiella pneumoniae bacteremia: influence of health care exposure on antimicrobial susceptibility. Diagn Microbiol Infect Dis 2006;55:135-141.

3. Kreger BE, Craven DE, Carling PC, McCabe WR. Gram-negative bacteremia. III. Reassessment of etiology, epidemiology and ecology in 612 patients. Am J Med 1980;68:332-343.

4. Huh K, Kim J, Cho SY, et al. Continuous increase of the antimicrobial resistance among gram-negative pathogens causing bacteremia: a nationwide surveillance study by the Korean Network for Study on Infectious Diseases (KONSID). Diagn Microbiol Infect Dis 2013;76:477-482.

5. Chung DR, Lee SS, Lee HR, et al. Emerging invasive liver abscess caused by $\mathrm{K} 1$ serotype Klebsiella pneumoniae in
Korea. J Infect 2007;54:578-583.

6. Jung Y, Lee MJ, Sin HY, et al. Differences in characteristics between healthcare-associated and community-acquired infection in community-onset Klebsiella pneumoniae bloodstream infection in Korea. BMC Infect Dis 2012;12:239.

7. Wie SH, Chang UI, Kim JD, et al. Clinical features of 141 cases of pyogenic liver abscess over a 10-year period and antibiotic sensitivity to the causative organisms. Infect Chemother 2008;40:199-206.

8. Garcia de la Torre M, Romero-Vivas J, Martinez-Beltran J, Guerrero A, Meseguer M, Bouza E. Klebsiella bacteremia: an analysis of 100 episodes. Rev Infect Dis 1985;7:143-150.

9. Jang TN, Kuo BI, Shen SH, et al. Nosocomial gram-negative bacteremia in critically ill patients: epidemiologic characteristics and prognostic factors in 147 episodes. J Formos Med Assoc 1999;98:465-473.

10. Sligl W, Taylor G, Brindley PG. Five years of nosocomial Gram-negative bacteremia in a general intensive care unit: epidemiology, antimicrobial susceptibility patterns, and outcomes. Int J Infect Dis 2006;10:320-325.

11. Lee JA, Kang CI, Joo EJ, et al. Clinical and microbiological characteristics of healthcare-associated infections in community-onset Klebsiella pneumoniae bacteremia. Infect Chemother 2012;44:56-61.

12. Dellinger RP, Levy MM, Rhodes A, et al. Surviving sepsis campaign: international guidelines for management of severe sepsis and septic shock: 2012. Crit Care Med 2013;41:580-637.

13. Lee JA, Kang CI, Joo EJ, et al. Epidemiology and clinical features of community-onset bacteremia caused by extended-spectrum $\beta$-lactamase-producing Klebsiella pneumoniae. Microb Drug Resist 2011;17:267-273.

14. Pirson M, Leclercq P, Jackson T, Leclercq M, Garrino M, Sion C. Financial consequences of hospital-acquired bacteraemia in three Belgian hospitals in 2003 and 2004. J Hosp Infect 2008;68:9-16. 